\title{
Gene Expression Profile of the Hypothalamus in DNP-KLH Immunized Mice Following Electroacupuncture Stimulation
}

\author{
Sun Kwang Kim, ${ }^{1,2,3}$ Jeungshin Kim, ${ }^{4}$ Eunjung Ko, ${ }^{1}$ Hyunseong Kim, ${ }^{1}$ Deok-Sang Hwang, ${ }^{5}$ \\ Sanghoon Lee, ${ }^{4}$ Yonghyeon Baek, ${ }^{4}$ Byung-Il Min, ${ }^{6}$ Sangsoo Nam, ${ }^{4}$ and Hyunsu Bae ${ }^{1,2}$ \\ ${ }^{1}$ Department of Physiology, College of Oriental Medicine, Kyung Hee University, \\ \#1 Hoegi-dong, Dongdaemoon-gu, Seoul 130-701, Republic of Korea \\ ${ }^{2}$ BK21 Oriental Medical Science Center, Republic of Korea \\ ${ }^{3}$ Acupuncture \& Meridian Science Research Center, Republic of Korea \\ ${ }^{4}$ Department of Acupuncture \& Moxibustion, College of Oriental Medicine, \\ Kyung Hee University, \#1 Hoeki-dong, Dongdaemoon-gu, Seoul, 130-701, Republic of Korea \\ ${ }^{5}$ Department of Oriental Gynecology, College of Oriental Medicine, Republic of Korea \\ ${ }^{6}$ Department of Physiology, College of Medicine, Kyung Hee University, Seoul, Republic of Korea \\ Correspondence should be addressed to Sangsoo Nam, dangun66@yahoo.co.kr and Hyunsu Bae, habae@khu.ac.kr
}

Received 5 August 2009; Accepted 25 November 2009

Copyright (c) 2011 Sun Kwang Kim et al. This is an open access article distributed under the Creative Commons Attribution License, which permits unrestricted use, distribution, and reproduction in any medium, provided the original work is properly cited.

\begin{abstract}
Clinical evidence indicates that electroacupuncture (EA) is effective for allergic disorder. Recent animal studies have shown that EA treatment reduces levels of IgE and Th2 cytokines in BALB/c mice immunized with 2,4-dinitrophenylated keyhole limpet protein (DNP-KLH). The hypothalamus, a brain center of the neural-immune system, is known to be activated by EA stimulation. This study was performed to identify and characterize the differentially expressed genes in the hypothalamus of DNP-KLH immunized mice that were stimulated with EA or only restrained. To this aim, we conducted a microarray analysis to evaluate the global gene expression profiles, using the hypothalamic RNA samples taken from three groups of mice: (i) normal control group (no treatments); (ii) IMH group (DNP-KLH immunization + restraint); and (iii) IMEA group (immunization + EA stimulation). The microarray analysis revealed that total 39 genes were altered in their expression levels by EA treatment. Ten genes, including T-cell receptor alpha variable region family 13 subfamily 1 (Tcra-V13.1), heat shock protein $1 \mathrm{~B}$ (Hspalb) and $2^{\prime}-5^{\prime}$ oligoadenylate synthetase $1 \mathrm{~F}$ (Oas1f), were up-regulated in the IMEA group when compared with the IMH group. In contrast, 29 genes, including decay accelerating factor $2(\mathrm{Daf} 2), \mathrm{NAD}(\mathrm{P}) \mathrm{H}$ dehydrogenase, quinone 1 (Nqo1) and programmed cell death 1 ligand 2 (Pdcd1lg2) were down-regulated in the IMEA group as compared with the IMH group. These results suggest that EA treatment can modulate immune response in DNP-KLH immunized mice by regulating expression levels of genes that are associated with innate immune, cellular defense and/or other kinds of immune system in the hypothalamus.
\end{abstract}

\section{Introduction}

Several clinical studies have previously suggested that electroacupuncture (EA) is effective for the treatment of various types of allergic disorders, including asthma and chronic urticaria [1-4]. It is generally accepted that hyperproduction of IgE in response to stimulation by Th2 cytokines (i.e., IL-4, IL-5 and IL-13) promotes the development of these allergic disorders $[5,6]$. Our previous study demonstrated that successive EA at the ST36 acupoint reduces serum levels of $\operatorname{IgE}$ in $\mathrm{BALB} / \mathrm{c}$ mice that have been immunized with 2,4-dinitrophenylated keyhole limpet protein (DNP-KLH) via suppression of the production of Th2 cytokines [7]. Furthermore, it has been shown that these effects of EA are mediated, at least in part, by $\alpha$-adrenergic receptors [8] and are acupoint (ST36) specific [9].

The hypothalamus is the primary center for neuroendocrine-immune modulation [10]. The paraventricular nucleus of the hypothalamus (PVN) represents an integral part of the neuroendocrine circuit that modulates immune function. Moreover, stimulation of the ventral nucleus of the hypothalamus $(\mathrm{VMH})$ stimulation enhances the immune 


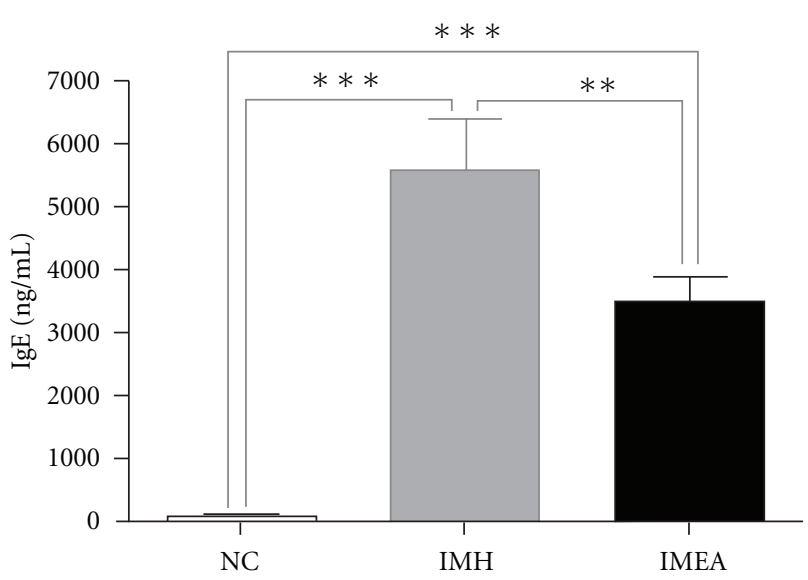

Figure 1: Comparison of serum IgE levels in the three experimental groups. The amounts of serum IgE were measured by ELISA. Data are presented as the mean \pm SEM. ${ }^{* *} P<.01,{ }^{* * *} P<.001$ between the two indicated groups as determined by the NewmanKeuls multiple comparison test following one-way ANOVA. NC ( $n=7$, no treatments for 2 weeks, except for i.p. injection of saline on the 1st and 8th experimental day); IMH ( $n=6$, immunization on the 1st and 8th experimental day + daily holder restraint); IMEA $(n=6$, immunization on the 1st and 8th experimental day+daily EA stimulation at the ST36 acupoint under holder restraint).

function associated with the lateral hypothalamus (LH) and ventral tegmental area (VTA) of the brain. Interestingly, previous brain imaging studies of animals and humans have shown that EA treatment affects the neuronal activity of the hypothalamus [11-16]. Although it has also been reported that EA influences the immunomodulatory effects of the hypothalamus $[17,18]$, the studies that have been conducted to date have only investigated the effects of EA on normal rats.

Oligonucleotide microarray analysis is the most powerful tool for functional genomics that provides direct information about mRNA expression levels from a large number of genes. This method has been used to find the genes correlated with the pathogenesis of diseases and to assess the drug actions on the diseases $[19,20]$. To examine if and how EA treatment modulates immune function of the hypothalamus at transcriptional level in a Th2-skewed condition, we conducted a microarray analysis on the hypothalamus of DNP-KLH immunized mice that were stimulated with EA.

\section{Methods}

2.1. Animals. Eight-week-old female BALB/c mice were purchased from Orient Bio (Sungnam, Korea) and housed in an air-controlled, pathogen-free animal facility under a 12h light/dark cycle at $23 \pm 2^{\circ} \mathrm{C}$. Mice were provided with a standard laboratory diet and water ad libitum. All procedures involving animals were conducted in accordance with the NIH guidelines.
2.2. Experimental Groups. Mice were randomly divided into the following 3 groups ( $n=6-10 /$ group): (i) NC group (normal control, no treatments for 2 weeks, except for i.p. injection of saline on the 1st and 8th experimental day); (ii) IMH group (immunization with 2,4-dinitrophenylated keyhole limpet protein (DNP-KLH) on the 1st and 8th experimental day and being held in a holder restraint for 20 min a day for 2 weeks; (iii) IMEA group (immunization on the 1st and 8th experimental day + daily EA stimulation at the ST36 acupoint while being held in a holder restraint for 20 min a day for 2 weeks).

2.3. EA Stimulation and DNP-KLH Immunization. EA stimulation was performed as previously described [7]. Briefly, a pair of stainless steel needles $(0.2 \mathrm{~mm}$ in diameter and $3 \mathrm{~cm}$ long) was inserted into the ST36, which is located at the anterior tibial muscle, $5 \mathrm{~mm}$ laterally and lower from the anterior tubercle of the tibia, and at a point $5 \mathrm{~mm}$ distal from the first needle. The anode and cathode leads from an electrical stimulator were then connected to the two acupuncture needles, after which train-pulses $(1 \mathrm{~Hz}, 0.25 \mathrm{~ms}$ pulse width, $3-5 \mathrm{~V}$ ) were applied for $20 \mathrm{~min}$. The stimulation intensity was determined to be the minimum voltage that induced a moderate muscle contraction.

All mice, except for the NC group, were immunized intraperitoneally with $4 \mu \mathrm{g}$ of DNP-KLH (Calbiochem, Gibbstown, NJ, USA) and $4 \mathrm{mg}$ of aluminum hydroxide (Sigma, St Louis, MO, USA) on the 1st and 8th day during the 2week experimental period. Immunization of $\mathrm{BALB} / \mathrm{c}$ mice with DNP-KLH caused a significant increase in $\operatorname{IgE}$ and IL-4 production on the 14th day after first immunization; therefore, EA stimulation was conducted daily for 2 weeks [7].

2.4. Measurements of IgE in Serum Using ELISA. Serum was obtained from all groups of mice on the final experimental day and then stored at $-20^{\circ} \mathrm{C}$ until analysis. The Serum level of IgE was measured using a quantitative sandwich enzyme-linked immunoassay kit (BD, Franklin Lakes, NJ, USA). Briefly, a 96-well microtiter plate (Coster, Cambridge, MA, USA) was incubated overnight at $4^{\circ} \mathrm{C}$ with antimouse IgE monoclonal antibody in coating buffer. The plate was then washed with PBS containing $0.05 \%$ tween 20 (Sigma, USA) and blocked with 5\% FBS in PBS for $1 \mathrm{~h}$ at room temperature. Subsequently, $100 \mu \mathrm{l}$ of sample were loaded onto the plate, which was then incubated for $2 \mathrm{~h}$ at room temperature. Next, secondary peroxidase labeled biotinylated anti-mouse IgE monoclonal antibody in 5\% FBS in PBS diluent was added and the plate was incubated for an additional $1 \mathrm{~h}$. The plates were then treated with TMB substrate solution for $30 \mathrm{~min}$, after which the reaction was stopped by the addition $50 \mu \mathrm{l}$ of $2 \mathrm{~N} \mathrm{H}_{2} \mathrm{SO}_{4}$ stop solution per well. Finally, the optical density at $450 \mathrm{~nm}$ was measured in a microplate reader (TECAN, Durham, NC, USA).

2.5. Oligonucleotide Chip Microarray. The hypothalamuses were obtained from all groups of mice on the final experimental day and then stored at $-80^{\circ} \mathrm{C}$ until analysis. 

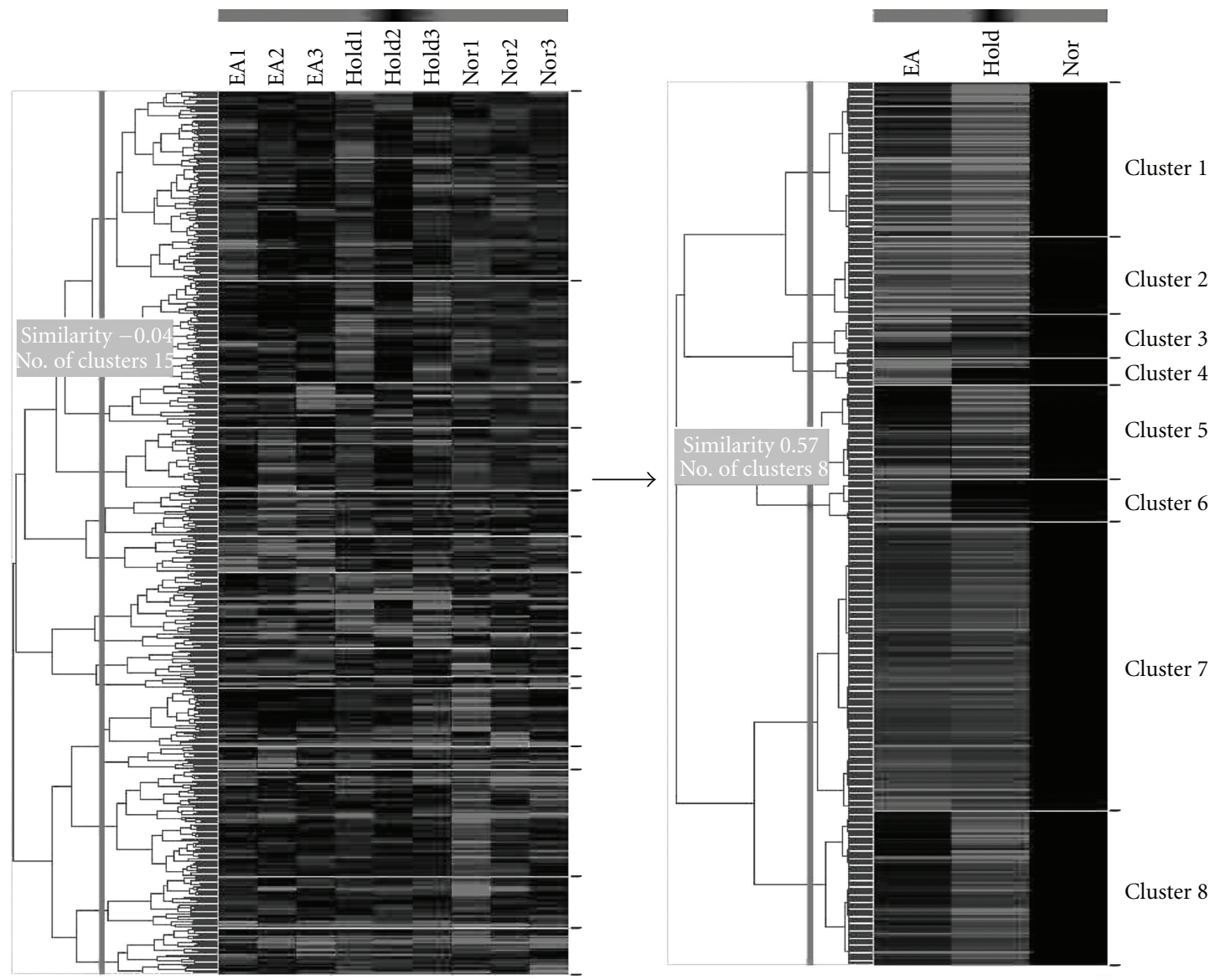

Figure 2: Clustergram of up- and down-regulated genes in DNP-KLH immunized BALB/c mice that were subjected to EA stimulation. The results shown are representative of three independent samples collected for each treatment. Each gene is represented by a single row of clustered boxes and each experimental sample is represented by a single column. The entire clustered image is shown on the left. The signal averages of up- and down-regulated genes are shown on the right.

Total RNA was extracted from the hypothalamus by using TRIZOL reagent, and DNase I (Invitrogen Life Technologies, Rockville, MD, USA) was treated to eliminate genomic DNA contamination. Oligonucleotide chip microarray was performed using single round RNA amplification protocols, following the Affymetrix specifications (Affymetrix GeneChip Expression Analysis Technical Manual). Briefly, $3 \mu \mathrm{g}$ of total RNA was used to synthesize first-strand complementary DNA (cDNA) using oligonucleotide probes with 24 oligodT plus T7 promoter as primers (Proligo LLC, Boulder, CO, USA) and the Superscript Choice System (Life Technologies, Invitrogen, Milan, Italy). Following double-stranded cDNA synthesis, the products were purified by phenol-chloroform extraction, after which biotinylated antisense complimentary RNA (cRNA) was generated through in vitro transcription using a BioArray RNA High-Yield Transcript Labeling Kit (ENZO Life Sciences Inc., Farmingdale, NY, USA). Next, the biotinylated labeled cRNA was fragmented, and then $10 \mathrm{~g}$ of the total fragmented cRNA was hybridized to the GeneChip Mouse Gene 1.0 ST Array (P/N901178, Affymetrix Inc., Santa Clara, CA, USA). The Affymetrix Fluidics Station 400 was then used to wash and stain the chips, after which the nonhybridized target was removed. The samples were then incubated with a streptavidin-phycoerythrin conjugate to stain the biotinylated cRNA. Next, the staining was amplified using goat IgG as a blocking reagent and biotinylated antistreptavidin antibody (goat), followed by a second staining step using a streptavidin-phycoerythrin conjugate. The fluorescence was then detected using the Genechip System Confocal Scanner (Hewlett-Packard, Santa Clara, USA), and analysis of the data contained on each GeneChip was conducted using the GeneChip 3.1 software produced by Affymetrix using the default settings. Global scaling was then used to compare the chips, with all probe sets being scaled to a user-defined target intensity of 150 .

2.6. Data Analysis. One-way ANOVA followed by NewmanKeuls multiple comparison test was used for statistical analysis of the IgE data. In all cases, $P<.05$ was considered significant. Data are presented as the mean \pm SEM.

For analysis of the microarray data, the MAS5 algorithm was used to evaluate the expression signals generated by the Affymetrix Mouse Gene 1.0 ST Array. Global scaling normalization was then performed, after which the normalized data were log-transformed using base 2 . Next, a fold change 
TABLE 1: Alterations in gene expression in DNP-KLH immunized BALB/c mice that were treated with EA stimulation.

\begin{tabular}{|c|c|c|c|}
\hline \multirow{2}{*}{ Genes } & \multirow{2}{*}{ Symbol } & \multicolumn{2}{|c|}{ Regulation profile and average fold change } \\
\hline & & NC versus Hold & Hold versus EA \\
\hline \multicolumn{4}{|l|}{$<$ Cluster 3> } \\
\hline T-cell receptor alpha variable region family 13 subfamily 1 & Tcra-V13.1 & -0.35 & 0.88 \\
\hline Limb expression 1 homolog (chicken) & Lix 1 & -0.11 & 0.3 \\
\hline Trefoil factor 2 (spasmolytic protein 1$)$ & Tff2 & -0.12 & 0.31 \\
\hline Heat shock protein $1 \mathrm{~B}$ & Hspalb & -0.08 & 0.38 \\
\hline Olfactory receptor 1436 & Olfr1436 & -0.23 & 0.55 \\
\hline Olfactory receptor 1475 & Olfr1475 & -0.43 & 1.15 \\
\hline $2^{\prime}-5^{\prime}$ oligoadenylate synthetase $1 \mathrm{~F}$ & Oaslf & -0.23 & 0.34 \\
\hline Olfactory receptor 689 & Olfr689 & -0.13 & 0.35 \\
\hline Olfactory receptor 26 & Olfr26 & -0.10 & 0.39 \\
\hline Probasin & Pbsn & -0.44 & 0.80 \\
\hline \multicolumn{4}{|l|}{$<$ Cluster $5>$} \\
\hline Dermatopontin & Dpt & 0.15 & -0.29 \\
\hline Decay accelerating factor 2 & Daf2 & 0.17 & -0.30 \\
\hline Anterior gradient homolog 3 (Xenopus laevis) & Agr3 & 0.13 & -0.35 \\
\hline Secretagogin, EF-hand calcium binding protein & Scgn & 0.36 & -0.35 \\
\hline 3-oxoacyl-ACP synthase, mitochondrial & Oxsm & 0.22 & -0.32 \\
\hline Empty spiracles homolog 2 (Drosophila) & Emx2 & 0.48 & -0.45 \\
\hline Aldehyde dehydrogenase 3 family, member B1 & Aldh3b1 & 0.22 & -0.38 \\
\hline Cytochrome P450, family 2, subfamily c, polypeptide 38 & Сур2c38 & 0.27 & -0.79 \\
\hline Sulfide quinone reductase-like (yeast) & Sqrdl & 0.20 & -0.28 \\
\hline Olfactory receptor 1231 & Olfr1231 & 0.27 & -0.35 \\
\hline Myosin light chain 2, precursor lymphocyte-specific & Mylc2pl & 0.13 & -0.26 \\
\hline NLR family, pyrin domain containing $4 \mathrm{E}$ & Nlrp4e & 2.32 & -3.96 \\
\hline Olfactory receptor 617 & Olfr617 & 0.21 & -0.37 \\
\hline Olfactory receptor 544 & Olfr544 & 0.29 & -0.37 \\
\hline Carboxylesterase 2 & Ces2 & 0.09 & -0.28 \\
\hline $\mathrm{NAD}(\mathrm{P}) \mathrm{H}$ dehydrogenase, quinone 1 & Nqo1 & 0.17 & -0.28 \\
\hline Sodium channel, voltage-gated, type $\mathrm{X}$, alpha & Scn10a & 0.17 & -0.45 \\
\hline \multicolumn{4}{|l|}{$<$ Cluster 6> } \\
\hline Olfactory receptor 1393 & Olfr1393 & -0.1 & -0.47 \\
\hline Insulin-like growth factor 2 mRNA binding protein 1 & Igf2bp1 & 0.05 & -0.29 \\
\hline Cathepsin 3 & Cts3 & 0.06 & -0.39 \\
\hline Olfactory receptor 720 & Olfr720 & -0.19 & -0.27 \\
\hline Cell division cycle 25 homolog C (S. pombe) & Cdc25c & 0.05 & -0.27 \\
\hline Programmed cell death 1 ligand 2 & Pdcd1lg2 & 0.05 & -0.34 \\
\hline Olfactory receptor 1310 & Olfr1310 & 0.03 & -0.55 \\
\hline Group specific component & Gc & -0.02 & -0.32 \\
\hline G protein-coupled receptor, family $C$, group 5 , member D & Gprc5d & -0.07 & -0.37 \\
\hline Serum amyloid A 4 & Saa4 & -0.51 & -0.53 \\
\hline Vomeronasal 2, receptor 65 & Vmn2r65 & 0.05 & -0.53 \\
\hline Intestine specific homeobox & Isx & 0.05 & -0.29 \\
\hline
\end{tabular}

and a Welch $t$-test were applied to select the differentially expressed genes (DEGs) using a fold change threshold of 1.2 and a $P<.05$ to indicate significance. Each probe set used in the Affymetrix GeneChip produces a detection call, with $\mathrm{P}$ (present call) indicating good quality, M (marginal call) indicating intermediate quality and A (absent call) indicating relatively low reliability. Therefore, probe sets that resulted in A calls were removed to filter false positives. A volcano plot was used to better visualize and compare the two DEG methods. The 1.2-fold DEGs were clustered using the 


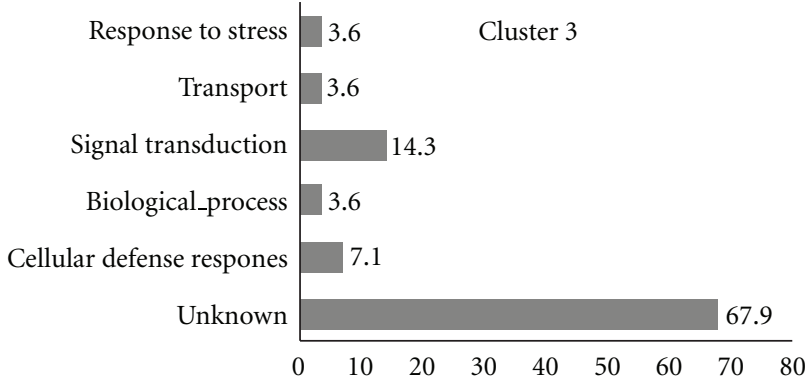

(a)

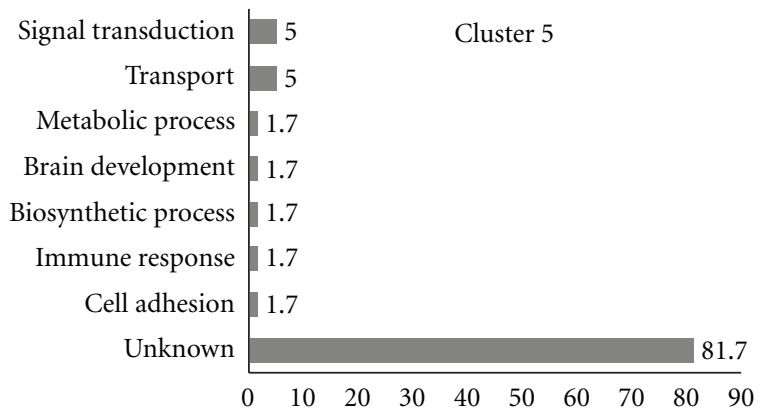

(b)

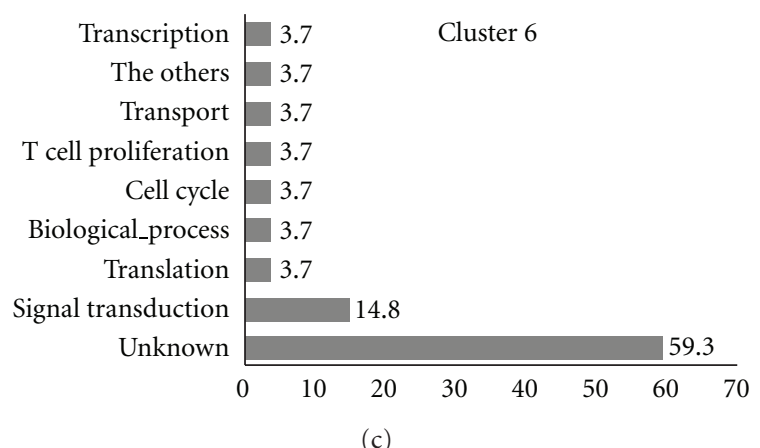

(c)

Figure 3: Gene ontology assignment of DEGs. Bars on Cluster 3 indicate up-regulated genes and bars on Cluster 5 and Cluster 6 indicate down-regulated genes comparing IMEA with IMH.

GenPlex $^{\mathrm{TM}}$ v2.3 software (ISTECH Inc., Ilsan, Korea) using hierarchical clustering with Pearson correlation as a similarity measure and complete linkage as the linkage method. In addition, gene ontology significance analysis was conducted to investigate the functional relationships among the 1.2fold DEGs using high-throughput GoMiner. The 1.2-fold DEGs were then mapped to the relevant pathways using the GenPlex v2.4 software (ISTECH Inc., Ilsan, Korea). The pathway resources were provided by the KEGG database.

\section{Results}

3.1. IgE Levels in DNP-KLH Immunized Mice Following EA Stimulation. BALB/c mice were immunized with DNP-KLH and aluminum hydroxide once a week. In the IMEA group, 20-min EA stimulation at the ST36 acupoint under restraint with an acryl holder was performed daily for 2 weeks, whereas mice in the IMH group were placed in the holder
TABLE 2: Categorized pathways based on comparison of gene expression between IMH and IMEA groups.

\begin{tabular}{|c|c|c|}
\hline KEGG Pathway & Gene counts & $P$-value \\
\hline $\begin{array}{l}\text { Metabolism of xenobiotics by } \\
\text { cytochrome P450 }\end{array}$ & 2 & $5.06 E-05$ \\
\hline Fatty acid biosynthesis & 1 & $1.18 E-03$ \\
\hline Biosynthesis of steroids & 1 & $3.87 E-03$ \\
\hline Phenylalanine metabolism & 1 & $5.22 E-03$ \\
\hline Histidine metabolism & 1 & $5.89 E-03$ \\
\hline Cell cycle & 1 & $5.95 E-03$ \\
\hline $\begin{array}{l}\text { Neuroactive ligand- } \\
\text { receptor interaction }\end{array}$ & 1 & $7.11 E-03$ \\
\hline Linoleic acid metabolism & 1 & $7.23 E-03$ \\
\hline Cell adhesion molecules (CAMs) & 1 & $7.69 E-03$ \\
\hline Tyrosine metabolism & 1 & $8.74 E-03$ \\
\hline Arachidonic acid metabolism & 1 & $1.13 E-02$ \\
\hline Complement and coagulation cascades & 1 & $1.18 E-02$ \\
\hline Hematopoietic cell lineage & 1 & $1.34 E-02$ \\
\hline Glycolysis/Gluconeogenesis & 1 & $1.38 E-02$ \\
\hline Leukocyte transendothelial migration & 1 & $1.79 E-02$ \\
\hline Tight junction & 1 & $2.17 E-02$ \\
\hline Focal adhesion & 1 & $3.25 E-02$ \\
\hline Regulation of actin cytoskeleton & 1 & $3.36 E-02$ \\
\hline
\end{tabular}

restraint for $20 \mathrm{~min}$ a day for 2 weeks, but not treated. After the 2-week experimental period, the serum levels of IgE were measured. Figure 1 shows serum levels of IgE in mice. The IgE levels in the IMH and the IMEA group were significantly increased when compared with the IgE levels of the NC group $(P<.001)$. However, EA treatment significantly reduced the elevated IgE levels $(P<.01$, IMEA versus IMH).

3.2. DEGs in the Hypothalamus of Mice in the NC, IMH and IMEA Groups. The genes that were differentially expressed among the groups are listed in Table 1 In addition, the genes are grouped into functional categories and pathways based on the KEGG database in Table 2. There were 10 genes that were down-regulated in the IMH group (versus $\mathrm{NC}$ ) and up-regulated in the IMEA group (versus IMH). Conversely, 17 genes were up-regulated in the IMH group (versus NC) and down-regulated in the IMEA group (versus IMH). Additionally, 12 genes were not significantly changed in the IMH group (versus NC), but were down-regulated in the IMEA group (versus IMH).

3.3. Clustering Analysis and Gene Ontology Analysis. As shown in Figure 2, microarray data from the normal control (NC) group and the experimental (IMH and IMEA) groups were combined and clustered using hierarchical clustering. To examine the functional relationships among the 1.2fold DEGs, the difference in gene ontology was determined using significance analysis (Figure 3 ). The results revealed that genes that were up- and down-regulated in the experimental groups were associated with processes including 


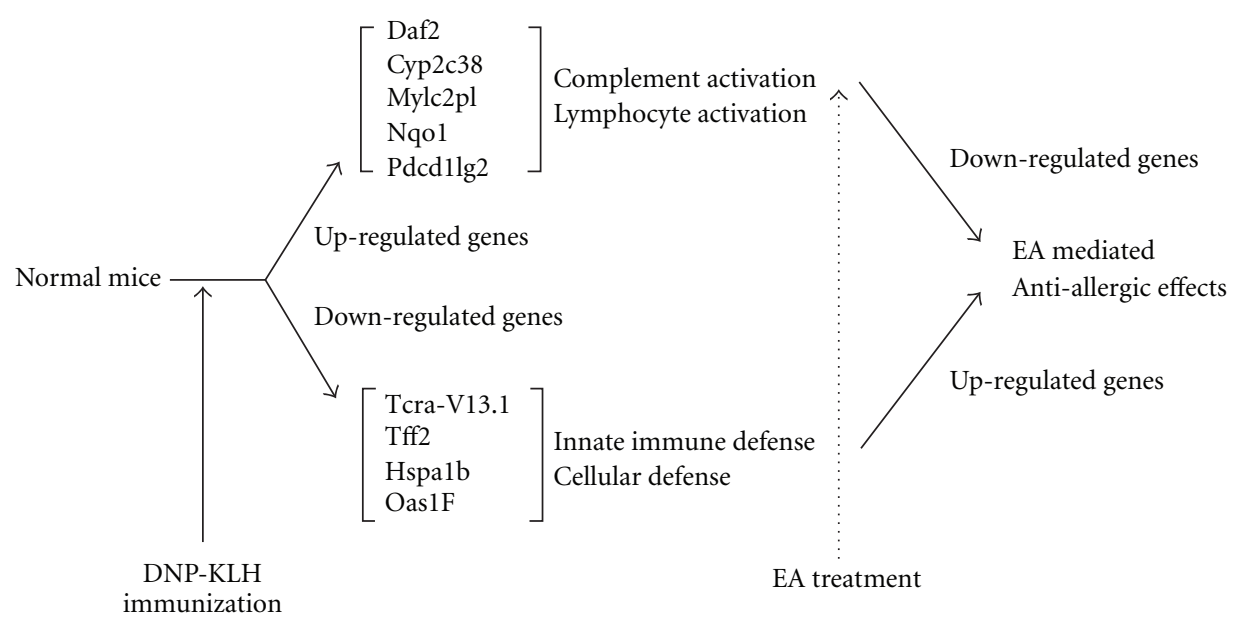

FIGURE 4: Summary of the mechanisms underlying EA-induced regulation of gene expression in the hypothalamus for anti-allergic effects.

signal transduction, cellular defense response, transport, transcription, biological processes, translation, the cell cycle and $\mathrm{T}$ cell proliferation.

\section{Discussion}

DNP-KLH immunization is known to induce the elevation of serum IgE levels and Th2 cytokines, which play important roles in the development of various allergic disorders [7,21]. In the present study, BALB/c mice in the IMH and IMEA groups were immunized with DNP-KLH and aluminum hydroxide. Although both groups showed a significant increase in IgE levels when compared with the NC group, IgE levels in the IMEA group were significantly lower than those in the IMH group (Figure 1). This result is consistent with the results of previous studies [7-9] and strongly suggests that EA treatment can modulate the immune response in the Th2 dominant condition.

Microarray analysis has been shown to be useful for the simultaneous profiling of global gene expression and identification of new genes or new functions of known genes [19, 20]. As described in the introduction, the hypothalamus is a primary center in the central nervous system responsible for neural-immune modulation [10] and has been shown to be affected by EA treatment $[11,12,15]$. Moreover, our recent study [18] showed that EA stimulation at ST36 can alter the transcriptional activity of the hypothalamus in normal rats. Therefore, we conducted microarray analysis of the hypothalamus of DNP-KLH immunized mice to determine if EA treatment of mice in a Th2-skewed condition can regulate gene expression in the hypothalamus.

Ten genes, including T-cell receptor alpha variable region family 13 subfamily 1 (Tcra-V13.1), trefoil factor 2 (spasmolytic protein 1) (Tff2), heat shock protein 1B (Hspalb) and $2^{\prime}-5^{\prime}$ oligoadenylate synthetase $1 \mathrm{~F}$ (Oas1f), were downregulated in the IMH group when compared with the NC group and up-regulated in the IMEA group when compared with the IMH group. Tcra-V13.1 is a subfamily of the Tcell receptor $\mathrm{V} \alpha$ chain and contributes to the recognition of self-MHC molecules or peptides that has polymorphisms associated with diabetes $[22,23]$. The expression pattern of Tff2, which is a member of the newly discovered protective factor of gastrointestinal mucous, may be associated with gastric cancer [24]. Hspalb is involved in innate immunity and diverse biological processes including anti-apoptosis, response to stress and DNA repair $[25,26]$. Oaslf is a transcription unit of a family of interferon-induced antiviral proteins that may play a role in the host's innate defense mechanism against viral infection [27, 28]. The differential expression of these genes indicates that EA treatment may increase some activities of innate immune and cellular defense at the transcriptional level.

Conversely, 17 genes including decay accelerating factor 2 (Daf2), cytochrome P450, family 2, subfamily c, polypeptide 38 (Cyp2c38), myosin light chain 2, precursor lymphocytespecific (Mylc2pl), NAD (P)H dehydrogenase, and quinone 1 (Nqo1) were up-regulated in the IMH group when compared with the NC group and downregulated in the IMEA group when compared with the IMH group. In addition, 12 genes, including programmed cell death 1 ligand 2 (Pdcd1lg2), were downregulated in the IMEA group when compared with the IMH group, but not significantly changed in the IMH group when compared with the NC group. Daf2 is a glycosylphosphatidylinositol (GPI)-anchored membrane regulator that inhibits both the classical and alternative pathways of complement activation [29]. Cyp2c38 is a member of a superfamily of ubiquitous hemoproteins that metabolize a vast array of foreign chemicals as well as endogenous compounds [30]. Mylc2pl is a regulatory myosin light chain gene that is expressed specifically in precursor $\mathrm{B}$ and $\mathrm{T}$ lymphocytes and may play a role in lymphocyte development [31]. Nqo1 is a cytosolic enzyme that catalyzes the metabolic reduction of quinines and derivatives and is an endogenous factor in the regulation of immune response and autoimmunity [32]. Pdcdllg2 is a ligand for programmed cell death-1, which is a receptor that plays an inhibitory role in $\mathrm{T}$ cell activation and has a potential role in Th2-mediated diseases [33]. As shown in Table 2 and Figure 3, it appears 
that no major or specific biochemical pathways are regulated by EA treatment. However, the present data suggest that EA treatment widely regulates immune response in the hypothalamus at the transcriptional level.

In conclusion, EA treatment was found to reduce the serum levels of IgE that were elevated by DNP-KLH immunization in BALB/c mice. Furthermore, EA treatment altered the expression levels of 39 genes in the hypothalamus, many of which are involved in immune response (Figure 4). Taken together, these results suggest that EA treatment may induce immunomodulation by regulating gene expressions in the hypothalamus of DNP-KLH immunized mice.

\section{Funding}

The Kyung Hee University Research Fund in 2008 (KHU20080600).

\section{Acknowledgment}

S. K. Kim and J. Kim contribute equally to this work.

\section{References}

[1] W. Biernacki and M. D. Peake, "Acupuncture in treatment of stable asthma," Respiratory Medicine, vol. 92, no. 9, pp. 11431145, 1998.

[2] C.-J. Chen and H.-S. Yu, "Acupuncture treatment of urticaria," Archives of Dermatology, vol. 134, no. 11, pp. 1397-1399, 1998.

[3] J. Che, "The effect of acupuncture on serum IgE levels in patients with chronic urticaria," Journal of Traditional Chinese Medicine, vol. 26, no. 3, pp. 189-190, 2006.

[4] T. Takishima, S. Mue, G. Tamura, T. Ishihara, and K. Watanabe, "The bronchodilating effect of acupuncture in patients with acute asthma," Annals of Allergy, vol. 48, pp. 4449, 1982.

[5] E. Maggie, "The Th1/Th2 paradigm in allergy," Immunotechnology, vol. 3, pp. 233-244, 1998.

[6] J. A. Woodfolk, "Cytokines as a therapeutic target for allergic diseases: a complex picture," Current Pharmaceutical Design, vol. 12, no. 19, pp. 2349-2363, 2006.

[7] M.-B. Park, E. Ko, C. Ahn et al., "Suppression of IgE production and modulation of Th1/Th2 cell response by electroacupuncture in DNP-KLH immunized mice," Journal of Neuroimmunology, vol. 151, no. 1-2, pp. 40-44, 2004.

[8] Y. Lee, S. K. Kim, Y. Kim et al., "The $\alpha$-adrenoceptor mediation of the immunomodulatory effects of electroacupuncture in DNP-KLH immunized mice," Neuroscience Letters, vol. 423, no. 2, pp. 149-152, 2007.

[9] S. K. Kim, Y. Lee, H. Cho et al., "A parametric study on the immunomodulatory effects of electroacupuncture in DNPKLH immunized mice," Evidence-Based Complementary and Alternative Medicine, 2009.

[10] D. Wrona, "Neural-immune interactions: an integrative view of the bidirectional relationship between the brain and immune systems," Journal of Neuroimmunology, vol. 172, no. 1-2, pp. 38-58, 2006.

[11] J. H. Chiu, H. C. Cheng, C. H. Tai et al., "Electroacupunctureinduced neural activation detected by use of manganeseenhanced functional magnetic resonance imaging in rabbits,"
American Journal of Veterinary Research, vol. 62, pp. 178-182, 2001.

[12] J. H. Chiu, M. S. Chung, H. C. Cheng et al., "Different central manifestations in response to electroacupuncture at analgesic and nonanalgesic acupoints in rats: a manganese-enhanced functional magnetic resonance imaging study," Canadian Journal of Veterinary Research, vol. 67, pp. 94-101, 2003.

[13] J. C. Hsieh, C. H. Tu, F. P. Chen et al., "Activation of the hypothalamus characterizes the acupuncture stimulation at the analgesic point in human: a positron emission tomography study," Neuroscience Letters, vol. 307, pp. 105-108, 2001.

[14] K. K. S. Hui, J. Liu, N. Makris et al., "Acupuncture modulates the limbic system and subcortical gray structures of the human brain: evidence from fMRI studies in normal subjects," Human Brain Mapping, vol. 9, no. 1, pp. 13-25, 2000.

[15] V. Napadow, N. Kettner, J. Liu et al., "Hypothalamus and amygdala response to acupuncture stimuli in carpal tunnel syndrome," Pain, vol. 130, no. 3, pp. 254-266, 2007.

[16] M.-T. Wu, J.-C. Hsieh, J. Xiong et al., "Central nervous pathway for acupunture stimulation: localization of processing with functional MR imaging of the brain-preliminary experience," Radiology, vol. 212, no. 1, pp. 133-141, 1999.

[17] G. S. Choi, S. D. Oha, J. B. Han et al., "Modulation of natural killer cell activity affected by electroacupuncture through lateral hypothalamic area in rats," Neuroscience Letters, vol. 329, no. 1, pp. 1-4, 2002.

[18] S.-W. Rho, G.-S. Choi, E.-J. Ko et al., "Molecular changes in remote tissues induced by electro-acupuncture stimulation at acupoint ST36," Molecules and Cells, vol. 25, no. 2, pp. 178$183,2008$.

[19] K. Izuhara and H. Saito, "Microarray-based identification of novel biomarkers in asthma," Allergology International, vol. 55, no. 4, pp. 361-367, 2006.

[20] S. Thornton, D. Sowders, B. Aronow et al., "DNA microarray analysis reveals novel gene expression profiles in collageninduced arthritis," Clinical Immunology, vol. 105, no. 2, pp. 155-168, 2002.

[21] H. Oka, Y. Emori, N. Kobayashi, Y. Hayashi, and K. Nomoto, "Suppression of allergic reactions by royal jelly in association with the restoration of macrophage function and the improvement of Th1/Th2 cell responses," International Immunopharmacology, vol. 1, pp. 521-532, 2001.

[22] E. A. Simone, Y. Liping, D. R. Wegmann, and G. S. Eisenbarth, "T cell receptor gene polymorphisms associated with antiinsulin, autoimmune T cells in diabetes-prone NOD mice," The Journal of Immunology, vol. 10, pp. 317-321, 1997.

[23] C. G. DiRienzo, G. F. Murphy, T. M. Friedman, and R. Korngold, "T-cell receptor V $\alpha$ usage by effector CD $4+\mathrm{V} \beta 11+$ $\mathrm{T}$ cells mediating graft-versus-host disease directed to minor histocompatibility antigens," Biology of Blood and Marrow Transplantation, vol. 13, no. 3, pp. 265-276, 2007.

[24] S.-Q. Shi, J.-T. Cai, and J.-M. Yang, "Expression of trefoil factors 1 and 2 in precancerous condition and gastric cancer," World Journal of Gastroenterology, vol. 12, no. 18, pp. 31193122, 2006.

[25] S. D. Klein and B. Brüne, "Heat-shock protein 70 attenuates nitric oxide-induced apoptosis in RAW macrophages by preventing cytochrome c release," Biochemical Journal, vol. 362, no. 3, pp. 635-641, 2002.

[26] E. Karoly, A. Fekete, N. F. Banki et al., "Heat shock protein 72 (HSPA1B) gene polymorphism and toll-like receptor (TLR) 4 mutation are associated with increased risk of urinary tract infection in children," Pediatric Research, vol. 61, no. 3, pp. 371-374, 2007. 
[27] S. Eskildsen, R. Hartmann, N. O. Kjeldgaard, and J. Justesen, "Gene structure of the murine 2' -5 '-oligoadenylate synthetase family," Cellular and Molecular Life Sciences, vol. 59, no. 7, pp. 1212-1222, 2002.

[28] T. Mashimo, P. Glaser, M. Lucas et al., "Structural and functional genomics and evolutionary relationships in the cluster of genes encoding murine $2^{\prime}, 5^{\prime}$-oligoadenylate synthetases," Genomics, vol. 82, no. 5, pp. 537-552, 2003.

[29] X. Sun, C. D. Funk, C. Deng, A. Sahu, J. D. Lambris, and W. C. Song, "Role of decay-accelerating factor in regulating complement activation on the erythrocyte surface as revealed by gene targeting," Proceedings of the National Academy of Sciences of the United States of America, vol. 96, pp. 628-633, 1999.

[30] G. Luo, D. C. Zeldin, J. A. Blaisdell, E. Hodgson, and J. A. Goldstein, "Cloning, and expression of murine CYP2Cs and their ability to metabolize arachidonic acid," Archives of Biochemistry and Biophysics, vol. 357, pp. 45-57, 1998.

[31] E. M. Oltz, G. D. Yancopoulos, M. A. Morrow et al., "A novel regulatory myosin light chain gene distinguishes pre-B cell subsets and is IL-7 inducible," The EMBO Journal, vol. 11, pp. 2759-2767, 1992.

[32] K. Iskander, J. Li, S. Han, B. Zheng, and A. K. Jaiswal, "NQO1 and NQO2 regulation of humoral immunity and autoimmunity," Journal of Biological Chemistry, vol. 281, no. 41, pp. 30917-30924, 2006.

[33] E. Oflazoglu, D. A. Swart, P. Anders-Bartholo et al., "Paradoxical role of programmed death-1 ligand 2 in Th2 immune responses in vitro and in a mouse asthma model in vivo," European Journal of Immunology, vol. 34, no. 12, pp. 33263336, 2004. 


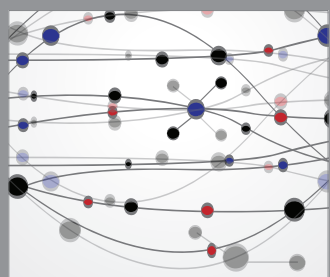

The Scientific World Journal
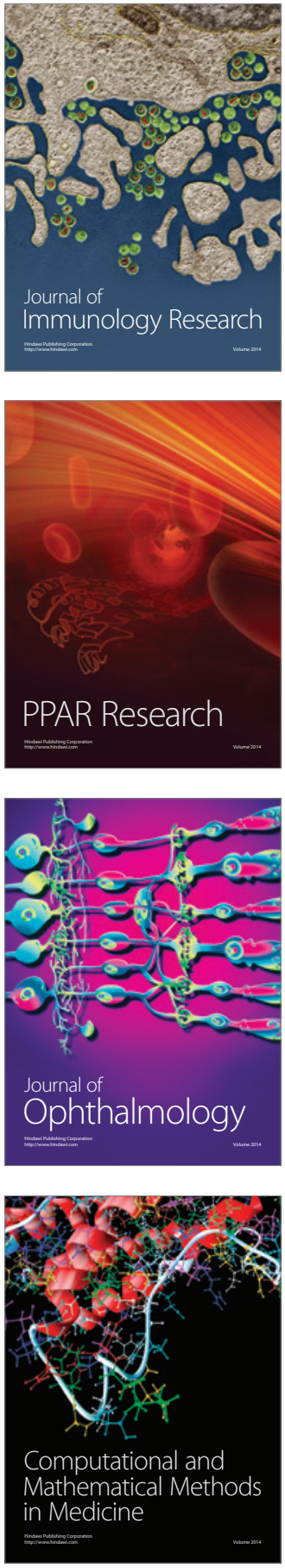

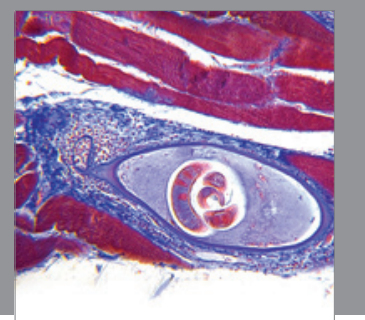

Gastroenterology

Research and Practice
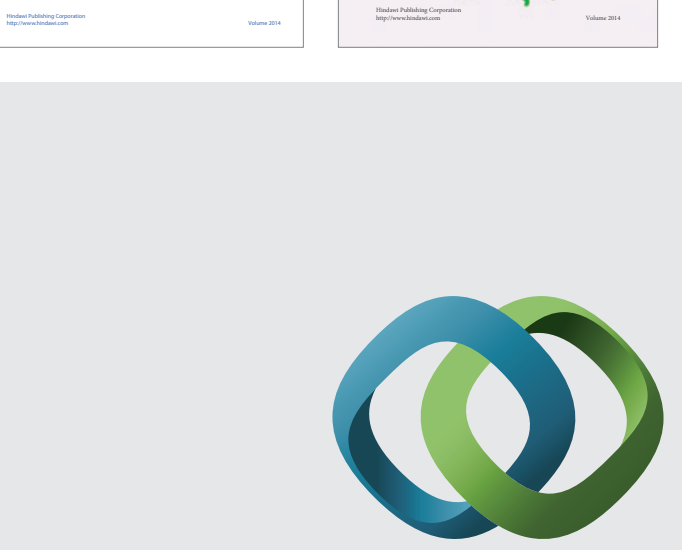

\section{Hindawi}

Submit your manuscripts at

http://www.hindawi.com
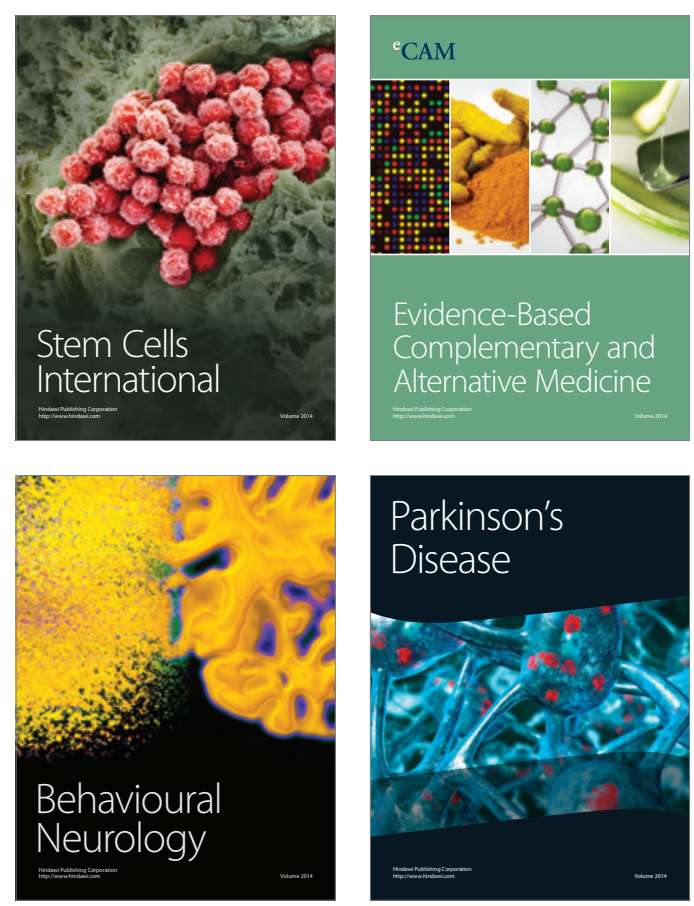

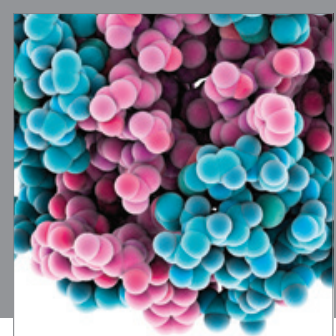

Journal of
Diabetes Research

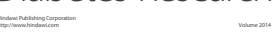

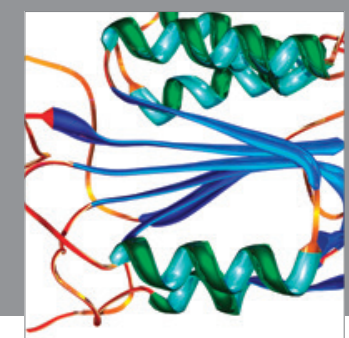

Disease Markers
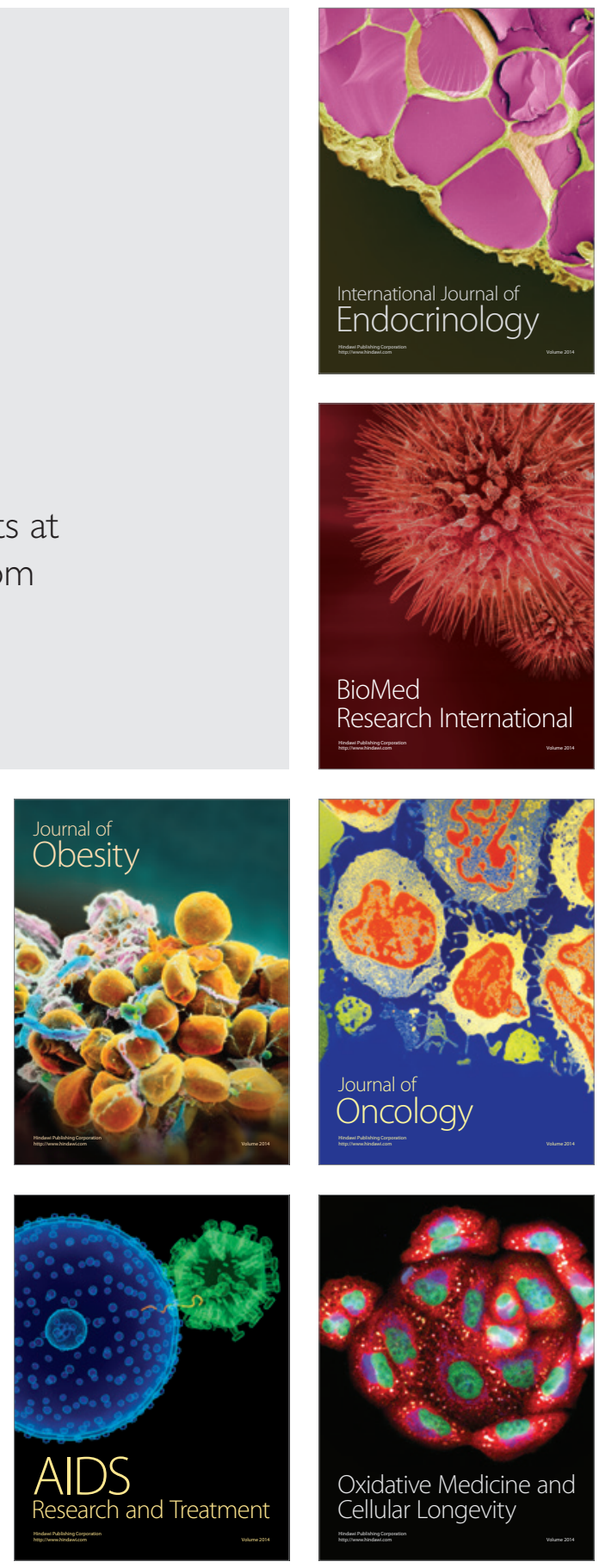